\title{
Электронно-микроскопические исследования распределения пор по диаметрам на сорбционной поверхности ультрафильтрационных мембран УАМ-50, УАМ-100, УПМ-К, УПМ-100
}

\author{
(C) 2020 Лазарев С.И. ${ }^{1}$, Ковалев С.В. ${ }^{1,3}$, Коновалов Д.Н. ${ }^{1}$, Рыжкин В.Ю. ${ }^{1}$, \\ Полянский К.К. ${ }^{2}$, Кореньков В.В. ${ }^{3}$, Хорохорина И.В. ${ }^{1}$, Ковалева О.А. ${ }^{1,3}$ \\ ${ }^{1}$ Тамбовский государственный технический университет, Тамбов \\ ${ }^{2}$ Воронежский филиал Российского экономического университета им. Г.В. Плеханова, Воронеж \\ ${ }^{3}$ Тамбовский государственный университет им. Г.Р. Державина, Тамбов
}

Поступила в редакцию 11.03.2020 г.

DOI: $10.17308 /$ sorpchrom.2020.20/3138

В работе обоснована актуальность заявленной тематики, определено место работы среди других публикаций в области исследования пористости мембран. На основе литературного обзора изучаемой области и сформулированной цели проведен выбор исследуемых типов ультрафильтрационных мембран, представлены их основные характеристики (рабочее давление, удельный поток растворителя, коэффициент задерживания, рабочий диапазон $\mathrm{pH}$, максимальная температура). Применена методика электронно-микроскопического исследования распределения пор по диаметрам на поверхности ультрафильтрационных мембран УАМ-50, УАМ-100, УПМ-К, УПМ-100, включающая в себя фиксацию изображения поверхности мембран с помощью электронного микроскопа, перенос в среду автоматизированного проектирования AutoCad 2018, визуальный выбор четырех равномерных зон, площадью по $1 \cdot 10^{6} \mathrm{Hм}^{2}$ с описыванием окружностью пор при использовании программы, экспорт данных с вычислением радиальных размеров на поверхностном (активном) слое мембраны в нм. Посредством програмной функции AutoCad 2018 находяться главные характеристики (диаметр, площадь каждого элемента), затем определялся коэффициент засоренности. Для оценки погрешности определения размеров пор анализировали 5 электронных изображений, полученных для различных участков поверхности исследуемой мембраны. Процедуру обработки каждого электронного изображения повторяли 10 раз. В результате статистической обработки результатов относительное стандартное отклонение не превышало 0.1. С помощью функции описательной статистики программой Microsoft Excel 2010 определялись среднеквадратичное отклонение, средний диаметр пор и производилось построение гистограмм, графиков функции плотности распределения вероятностей и нормального распределения для исследуемых мембран от распределения диаметров пор.

Установлено, что распределение пор по диаметрам на поверхности активного слоя исследуемых ультрафильтрационных мембран описывается законом нормального распределения (закон Гаусса). Получены эмпирические выражения функции нормального распределения пор по диаметрам. Анализ экспериментальных исследований методом электронной микроскопии, стандартных средств обработки данных Microsoft Excel 2010, AutoCad 2018 показал, что для образцов мембран УАМ-50, УАМ-100, УПМ-К, УПМ-100 средний диаметр пор на поверхности находится в интервале от 54 до 70 нм.

Ключевые слова: диаметр микроструктурных неоднородностей, электронно-микроскопическое, функция нормального распределения, методика, гистограмма. 


\section{Введение}

Предприятия машиностроения, химической промышленности и т. П. для очистки технологических растворов и сточных вод используют мембранные процессы разделения, но проблема использования мембран с высокими показателями по качеству очистки от растворенных веществ и постоянной удельной производительностью во времени всегда остается актуальной [1]. Процессы мембранного разделения растворов, особенно биологических и пищевых жидкостей, сопровождаются осадко- и гелеобразованием, а также специфическим взаимодействием компонентов водной среды с поверхностью мембран. В некоторой иностранной литературе подобные явления связывают с эффектом обрастания мембран [1-7].

Массопереносные и сорбционные характеристики мембран [8-11] зависят от многих факторов (трансмембранное давление, перепад электрического потенциала на мембране и др.), в частном случае, подобные характеристики связаны и с проницаемостью компонентов раствора через поверхность мембран, а это также зависит от распределения пор по радиусам для конкретного типа мембран. Разработка перспективных методик оценки распределения пор по радиусам и микроструктуры поверхности для той или иной мембранной перегородки является актуальной.

Работа [12] посвящена разработке пакета прикладных программ обработки цифровых изображений для расчета пористости, распределения диаметра, площади, формы пор на поверхности мембраны путем обработки снимков, полученных при помощи сканирующей электронной микроскопии, по сравнению с результатами анализа прибором IBAS I/II (Германия) исследованная пористость больше, а дисперсия распределения шире.

Проблемы и перспективы применения метода ультрафильтрации в разделении растворов и очистке воды описаны в работе [13]. Отмечается, что размер отверстий (пор) ультрафильтрационных мембран находится в пределах от 5 нм до 0.05-0.1 мкм. Авторами работы [14] предлагается новый и эффективный тест по определению размера пор, основанный на синтезе и переносе через мембрану жестких наночастиц. Монодисперсии золота и серебра 3-50 нм обеспечили полное распределение пор по размерам, включая диаметр пор, при котором мембрана имеет $100 \%$ удерживающую способность (d100). Значения d100 в тестируемых УФ-мембранах варьируются от 40 нм до 50 нм в зависимости от материала мембраны. В работе [15] представлен тест по измерению размера пор мембраны ультрафильтрации быстрым, чувствительным и надежным способом. Мембраны для ультрафильтрации, изготовленные из различных полимерных материалов, подвергали испытанию смесью частиц золота размером 20 , 30,40 и 60 нм в концентрации приблизительно $7 \times 10^{7}$ частиц $/ \mathrm{cm}^{3}$ для каждого номинального размера или $2,8,19,66$ мкг/дм ${ }^{3}$ золото, соответственно, для определения эффективности фильтрации в широком диапазоне размеров. Тест на удерживание показал, что две самые плотные мембраны из пяти протестированных достигли полного удержания частиц диаметром от 40 до 60 нм.

Эксперименты по ультрафильтрации с белковыми растворами и мембранами с различными измеренными потоками воды подтверждают, что гель-поляризованный поток зависит от проницаемости мембраны и свойств поверхности, а значит и от распределения пор по поверхности [16]. В работе [17] была использована модифицированная мембрана на основе $\mathrm{N}$-акрилоилморфолина (АСМ), которая показала максимальную пористость, плотность пор и проницаемость при 1.5 мас.\% сополимера. Авторами [18] разработана модель проницаемости-селективности, которая позволяет выявить соотношения размеров пор и их распределения по 
размерам на удовлетворительную проницаемость и селективность мембраны. В работе [19] изготовлены и исследованы две асимметричные мембраны из ацетата целлюлозы М1 и М2 со средним радиусом пор 2.6 и 5.3 нм, соответственно. Водные растворы лизоцима, содержащие $0,1 \mathrm{M} \mathrm{NaCl}$, подвергали ультрафильтрации через мембраны M1 и М2. Прогнозируемое задерживание лизоцима с учетом развития двойных электрических слоев на поверхности пор белка и мембраны хорошо согласуется с экспериментальными результатами авторов.

В работе [20] проведены исследования по разработке модели нейронной сети для прогнозирования размера пор ультрафильтрационной мембраны. Обычно размер пор ультрафильтрационной мембраны определяли экспериментально, используя эксперименты со скоростью проникновения и отторжения с последующим составлением эмпирических уравнений. Искусственная нейронная сеть (ИНС) была предложена в качестве метода для прогнозирования размера пор плоских листовых ультрафильтрационных мембран. Результаты показали, что нейронные сети смогли точно оценить размер пор ультрафильтрационной мембраны. В статье [21] сообщается о применении двух различных искусственных нейронных сетей, многослойного персептрона и радиальной базовой функции по сравнению с обычным программным обеспечением для прогнозирования размера пор асимметричных полиэфирсульфоновых ультрафильтрационных мембран в качестве альтернативного метода. С развитием модели ИНС процесс оценки размера пор мембраны может быть упрощен и ускорен по сравнению с другими математическими решениями.

В литературе [22] обсуждается возможность использования данных калибровки белков для экспресс-анализа структуры ультрафильтрационной мембраны. Показано, что структурный анализ на основе данных калибровки дает возможность определить средний радиус пор мембраны и вид распределения пор по размерам, выявить и оценить микроскопическую дефектность мембраны, оценить толщину селективного слоя и также сопоставить структуру исследуемой мембраны со структурой основных серийных ультрафильтров.

Целью данной работы являются экспериментальные исследования распределения пор по диаметрам ультрафильтрационных мембран УАМ-50, УАМ-100, УПМ-К, УПМ-100.

\section{Экспериментальная часть}

Объектами исследования являлись ультрафильтрационные мембраны УАМ50, УАМ-100, УПМ-К, УПМ-100 производства ЗАО НТЦ «Владипор», г. Владимир, характеристики которых представлены в таблице 1.

Таблица 1. Характеристики ультрафильтрационных мембран УПМ и УАМ [23] Table 1. Characteristics of UPM and UAM ultrafiltration membranes [23]

\begin{tabular}{|c|c|c|c|c|c|}
\hline $\begin{array}{c}\text { Марка } \\
\text { проницае- } \\
\text { мых мембран }\end{array}$ & $\begin{array}{c}\text { Рабочее } \\
\text { давление } P, \\
\text { МПа }\end{array}$ & $\begin{array}{c}\text { Удельный } \\
\text { поток рас- } \\
\text { творителя } J, \\
\mathrm{M}^{3} / \mathrm{M}^{2} \mathrm{c}\end{array}$ & $\begin{array}{c}\text { Коэффици- } \\
\text { ент задержи- } \\
\text { вания по аль- } \\
\text { бумину } \\
67000 Д\end{array}$ & $\begin{array}{c}\text { Рабочий } \\
\text { диапазон } \\
\mathrm{pH}\end{array}$ & $\begin{array}{c}\text { Максималь- } \\
\text { ная темпера- } \\
\text { тура, }{ }^{\circ} \mathrm{C}\end{array}$ \\
\hline УАМ-50 & 0.15 & $3.33 \cdot 10^{-6}$ & 0.97 & $3-8$ & 50 \\
\hline УАМ-100 & 0.15 & $1.67 \cdot 10^{-6}$ & 0.97 & $3-8$ & 50 \\
\hline УПМ-К & 0.1 & $1.16 \cdot 10^{-5}$ & 0.98 & $2-12$ & 100 \\
\hline УПМ-100 & 0.1 & $2.33 \cdot 10^{-4}$ & 0.97 & $2-12$ & 100 \\
\hline
\end{tabular}


Мембраны типа УАМ представляют собой пористые полимерные полупрозрачные или белого цвета пленки на основе ацетатов целлюлозы. Ароматический полисульфонамид «Сульфон - 4Т» является основой мембран типа УПМ. В качестве подложки в обоих типах мембран используется нетканый лавсан или полипропилен.

Выбор в качестве объектов исследования пористых ультрафильтрационных мембран обеспечен высокой задерживающей способностью, хорошей производительностью и наибольшей применяемостью в промышленной практике.

Методика исследования состояла из следующих этапов. Заранее выбранные для эксперимента сухие образцы мембран УАМ-50, УАМ-100, УПМ-К, УПМ-100 подвергались исследованию (визуализации поверхности с помощью электронного микроскопа JEOLJSM 6510, НОЦ «Нанотехнологии и наноматериалы» ТГУ им. Г.Р. Державина), проводилась процедура фиксации изображения поверхности мембран (рис. 1). Затем данное изображение переносилось в точном соответствии с масштабом в среду автоматизированного проектирования AutoCad 2018, где происходила дальнейшая обработка изображения.

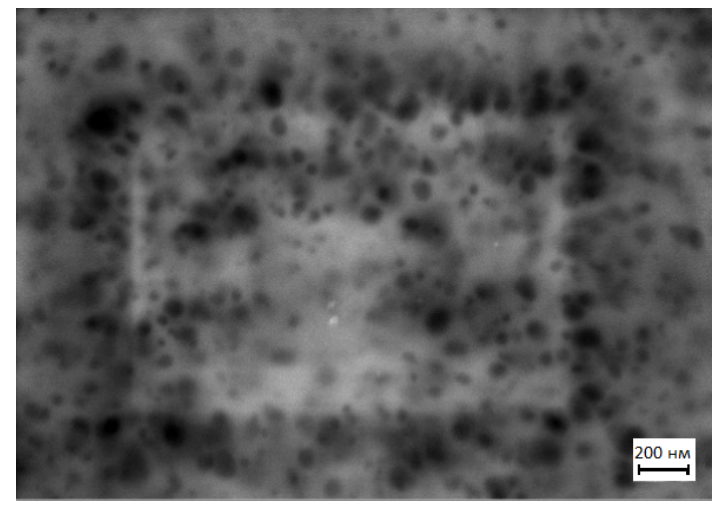

a

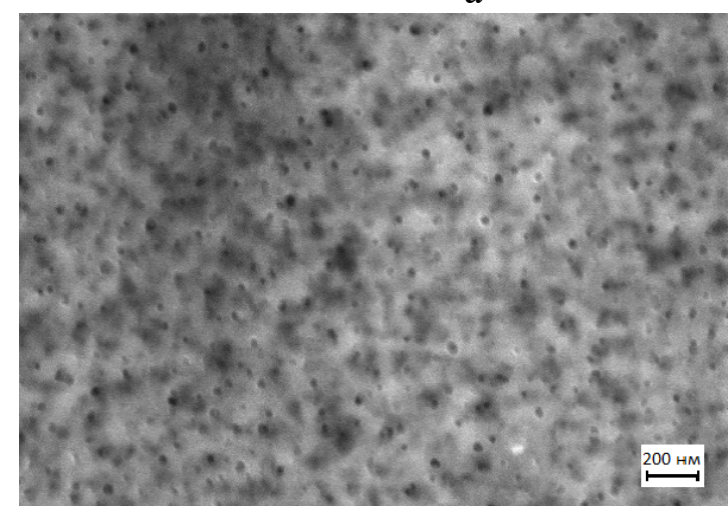

B

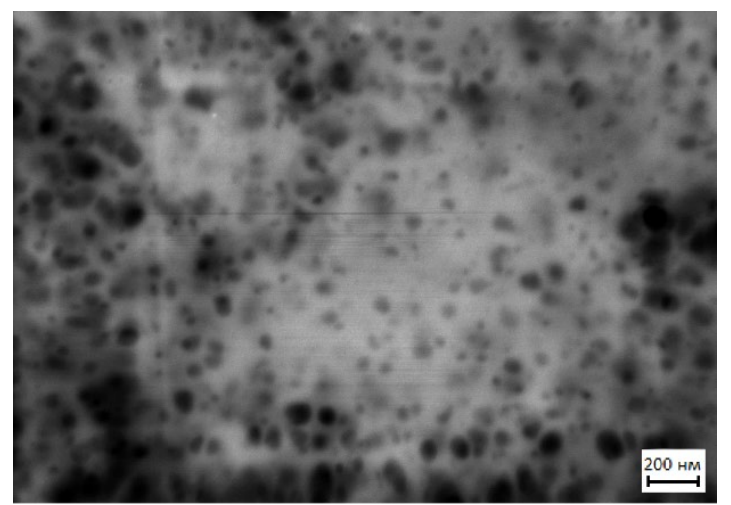

6

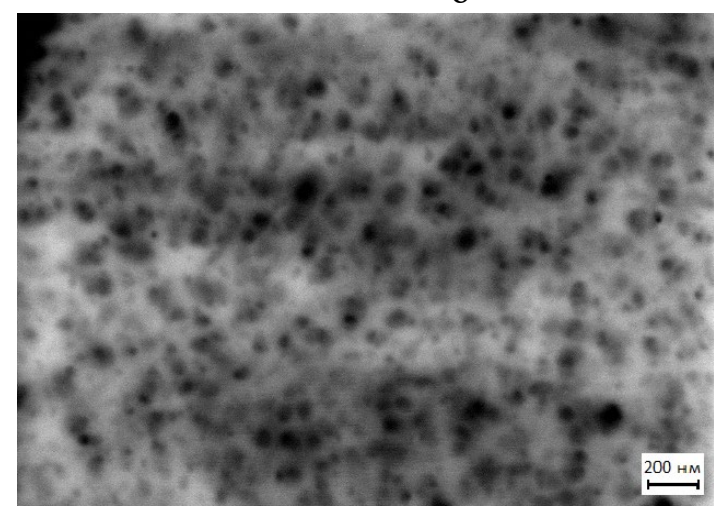

$\Gamma$

Рис. 1. Электронные изображения образцов ультрафильтрационных мембран УАМ-50 (а), УАМ-100 (б), УПМ-К (в), УПМ-100 (г) при увеличении 100 и ускоряющем напряжении 5 кВ

Fig. 1. Electronic images of UAM-50 (a), UAM-100 (b), UPM-K (c), UPM-100 (d) ultrafiltration membranes at a magnification of 100 with an accelerating voltage of $5 \mathrm{kV}$.

Сначала на полученных фотографиях поверхности мембран (рис. 2) визуально выбирались четыре равномерные зоны. Далее, как было установлено ранее, для каждой мембраны площадь выбранных квадратных зон составляла по $1 \cdot 10^{6}$ нм$^{2}$ соотвественно, где масштаб принятых областей строго соответствовал масштабу фотографий. Затем визуально определялись поры, которые описывались окружностью с помощью программы. После разметки пор проводилась процедура экспорта данных в систему 
автоматизированного проектирования AutoCad 2018 с помощью консольной команды «ДАННЫЕ ИЗВЛ». Посредством программы AutoCad 2018, предназначенной для автоматизированного проектирования, вычислялись радиальные размеры на поверхностном (активном) слое мембраны в нм.

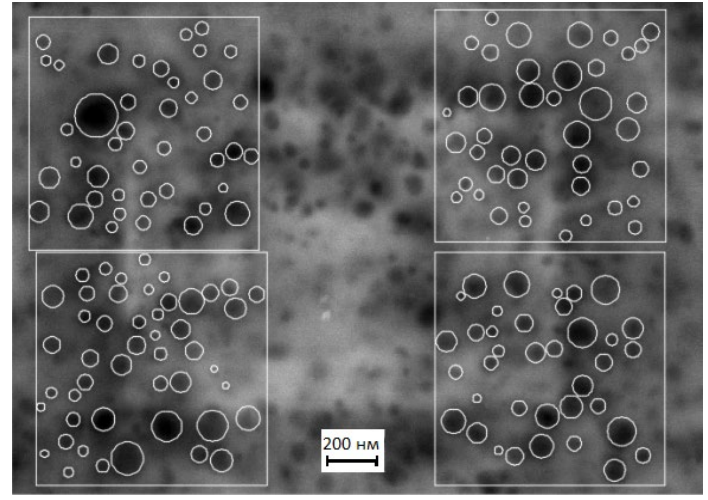

a

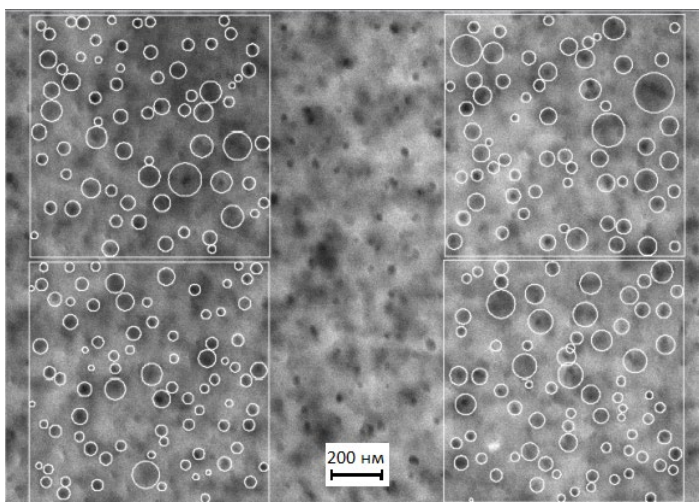

B

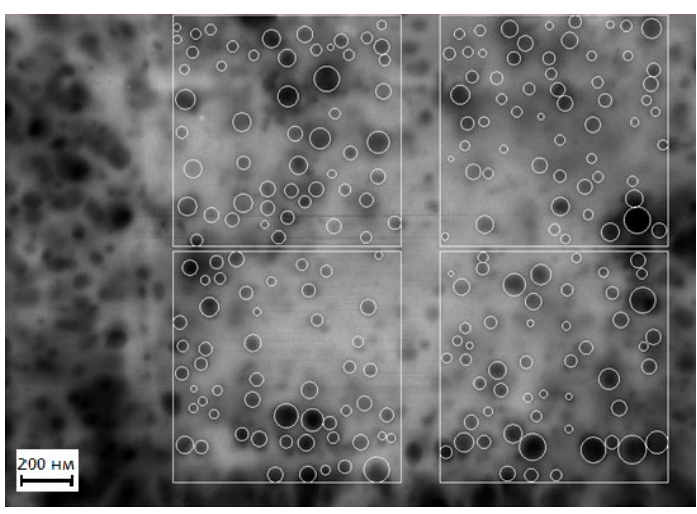

б

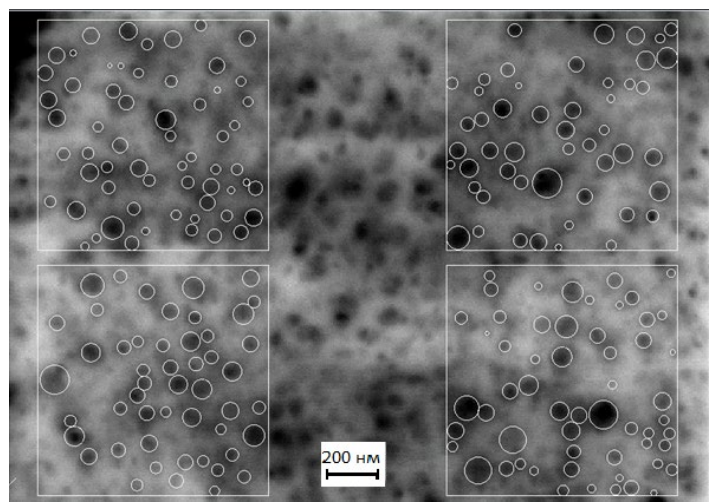

$\Gamma$

Рис. 2. Электронные изображения поверхности ультрафильтрационных мембран вида УАМ-50 (а), УАМ-100 (б), УПМ-К (в), УПМ-100 (г) с четырьмя равномерными зонами по периметру при увеличении 100 и ускоряющем напряжении 5 кВ

Fig. 2. Electronic images of the surface of UAM-50 (a), UAM-100 (b), UPM-K (c), UPM-100 (d) ultrafiltration membranes with four uniform zones around the perimeter at a magnification of 100 with an accelerating voltage of $5 \mathrm{kV}$

Посредством программной функции AutoCad 2018 «извлечение данных» находятся главные характеристики (диаметр, площадь каждого элемента) для всех четырёх квадратных областей выборки. Использование функции нахождения данных дало возможность производить анализ поверхности для каждой мембраны (таблица 2).

Для оценки погрешности определения размеров пор анализировали 5 электронных изображений, полученных для различных участков поверхности исследуемой мембраны. Процедуру обработки каждого электронного изображения повторяли 10 раз. В результате статистической обработки результатов относительное стандартное отклонение не превышало 0.1 .

Для расчета площади чистой поверхности квадратных областей-выборок производилось сложение площадей выборок, а далее вычитание их из общей области выборки. Затем определялся коэффициент засоренности по следующей расчетной формуле (1):

$$
K=\frac{S_{3}}{S_{\mathrm{u}}},
$$

где $S_{4}, S_{3}$ - площадь чистой и затемненной поверхности на мембране, нм$^{2}$. 
Полученные характеристики представлены в таблице 2.

Таблица 2. Расчетные площади на поверхности ультрафильтрационных мембран УПМ и УАМ

Table 2. Estimated areas on the surface of UPM and UAM ultrafiltration membranes

\begin{tabular}{|c|c|c|c|}
\hline Тип мембраны & $\begin{array}{c}\text { Площадь затемненной } \\
\text { поверхности } \mathrm{S}_{3}, \mathrm{HM}^{2}\end{array}$ & $\begin{array}{c}\text { Площадь чистой по- } \\
\text { верхности } \mathrm{S}_{\text {ч, }} \text { нм }^{2}\end{array}$ & $\begin{array}{c}\text { Коэффициент } \\
\text { засоренности, } \mathrm{K}\end{array}$ \\
\hline УАМ-50 & $0.74 \cdot 10^{6}$ & $3.26 \cdot 10^{6}$ & 0.23 \\
\hline УАМ-100 & $0.59 \cdot 10^{6}$ & $3.46 \cdot 10^{6}$ & 0.16 \\
\hline УПМ-К & $0.79 \cdot 10^{6}$ & $3.20 \cdot 10^{6}$ & 0.25 \\
\hline УПМ-100 & $0.65 \cdot 10^{6}$ & $3.35 \cdot 10^{6}$ & 0.16 \\
\hline
\end{tabular}

Для аналитической обработки полученных данных в результате исследований применялась программа Microsoft Excel 2010. С помощью функции описательной статистики программой определялись такие параметры как среднеквадратичное отклонение и средний диаметр пор.

Для проведения дальнейших расчетов возникает необходимость проверки правильности анализа полученных данных, которая решается при построении гистограмм. Построение гистограмм производится с применением стандартных настроек Microsoft Excel 2010. В программе выполняется автоматизированный выбор диапазона, полученных в ходе расчёта данных и производится построение гистограмм для мембран УАМ-50, УАМ-100, УПМ-К, УПМ-100.

При автоматизированном расчете ЭВМ самостоятельно выбирается размах, который определяется по расчетной формуле (2):

$$
R=X_{\max }-X_{\min },
$$

где $X_{\max }$ - максимальное значение выборки, $X_{\min }$ - минимальное значение выборки.

Этот параметр (размах) показывает, какой будет ширина гистограммы и определяется разброс полученных величин. Затем ЭВМ разбивает полученный диапазон на несколько интервалов, количество которых рассчитывается по формуле (3):

$$
k=\sqrt{n \pm 2},
$$

где $n$ - коэффициент, учитывающий количество найденных значений диаметров выборки.

Затем посредством электронно-вычислительной машины рассчитывалась ширина интервала $(h)$, которая определяется из формулы (4):

$$
h=\frac{R}{k} \text {. }
$$

Далее производится построение функции нормального распределения диаметров пор мембран.

\section{Обсуждение результатов}

Результаты количественной оценки среднего размера пор на сорбционной поверхности исследуемых ультрафильтрационных мембран представлены в таблице 3. Установлено, что средний диаметр пор мембран УАМ-100, УПМ-К, УПМ-100 находится в диапазоне от 54 до 59 нм. Средний размер пор на поверхности мембраны УАМ-50 составляет $70 \pm 4$ нм. 
На рис. 3 представлены гистограммы распределения пор по диаметрам на поверхности исследуемых образцов ультрафильтрационных мембран. Кривые распределения свидетельствуют о появлении пор размером более 130 нм на поверхности мембраны УАМ-50 по сравнению с мембранами УАМ-100 и УПМ.

Таблица 3. Средний диаметр пор и их среднеквадратичное отклонение Table 3. Average pore diameter and their standard deviation

\begin{tabular}{|c|c|c|}
\hline Тип мембраны & $\begin{array}{c}\text { Средний диаметр пор } \\
\mathrm{d}_{\text {cp }}, \text { нм }\end{array}$ & $\begin{array}{c}\text { Среднее квадратичное } \\
\text { отклонение, } \sigma\end{array}$ \\
\hline УАМ-50 & $70 \pm 4$ & 1.97 \\
\hline УАМ-100 & $58 \pm 3$ & 1.44 \\
\hline УПМ-К & $54 \pm 3$ & 1.21 \\
\hline УПМ-100 & $59 \pm 3$ & 1.33 \\
\hline
\end{tabular}

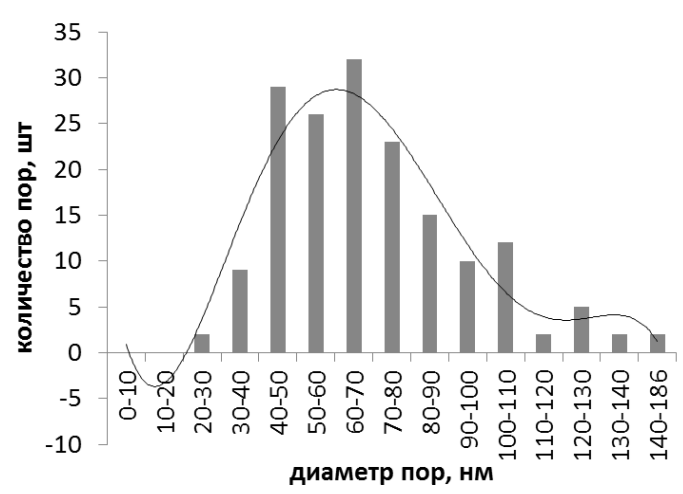

a

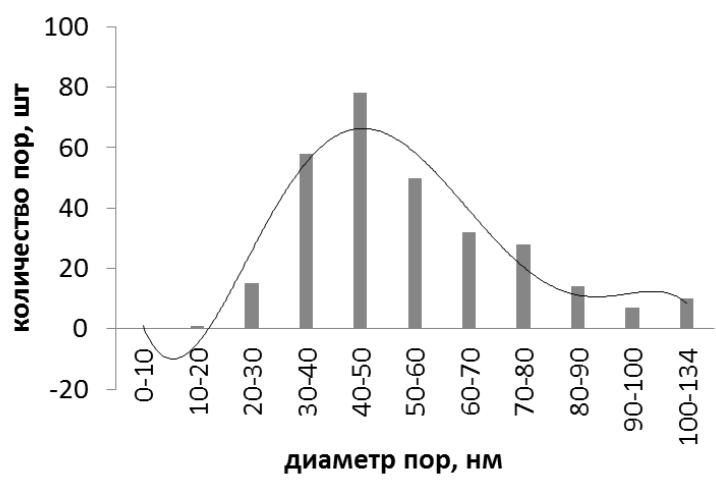

B

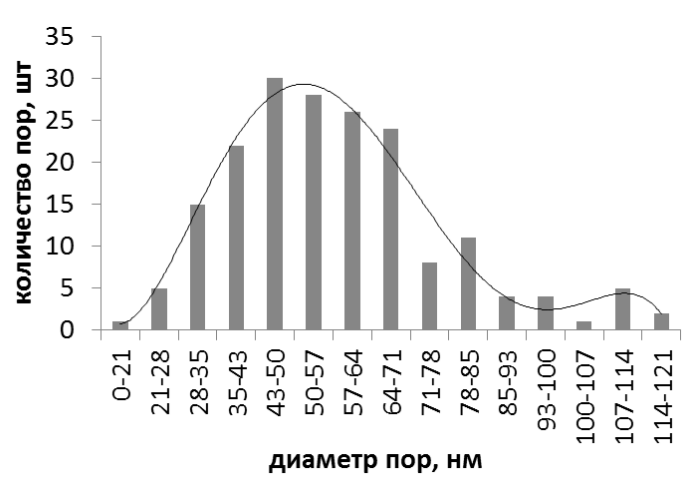

6

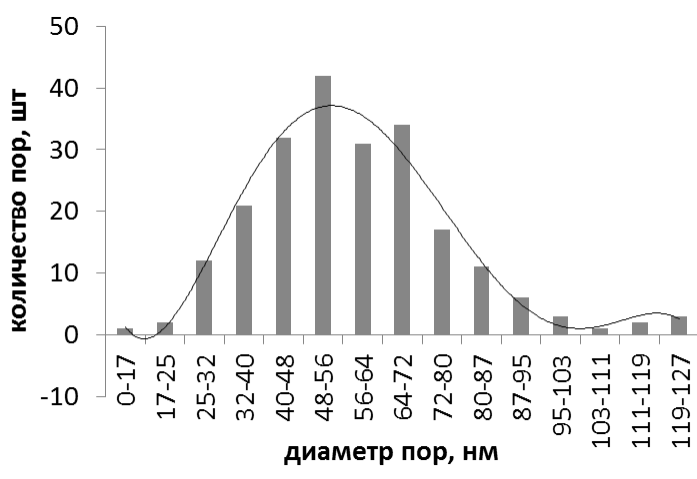

$\Gamma$

Рис. 3. Гистограммы распределения пор по диаметрам на поверхности мембран УАМ-50 (а), УАМ-100 (б), УПМ-К (в), УПМ-100 (г)

Fig. 3. Histograms of distribution of pores by diameters on the surface of UAM-50 (a), UAM-100 (b), UPM-K (c), UPM-100 (d) membranes

Построенные гистограммы распределения пор по диаметрам на поверхности мембран УАМ-50, УПМ-100 можно ошибочно отнести к мультимодальному типу (рис. 3 а, г), но при сглаживании (осреднении) пиковых значений распределения пор по диаметрам линией тренда полиномиального типа 5 порядка отмечается схожесть ее с гистограммой обычного (колоколообразного) типа, как и в случае гистограмм для мембран УАМ-100 и УПМ-К (рис. 3 б, в). Сглаживание гистограмм, представленных на рис. 3 (а-г) для исследуемых образцов мембран УАМ-50, УАМ-100, УПМ-К, 
УПМ-100 произведено из-за допущения о том, что поры описываются формой окружности. Это в некоторой степени объясняет наличие пиковых значений частот исследуемых значений, представленных на гистограмме (так называемое наличие доминирующей погрешности (специальной причины)) [24].

Полученные экспериментальные данные по средним размерам пор ультрафильтрационных мембран для чистых поверхностей (не прошедших обработку в экспериментальной ячейке под давлением при ультрафильтрации) сопоставимы с данными работы [25] по размерам пор синтезированных ультрафильтрационных мембран, а также данными из литературы [12-15].

Графики функции плотности распределения вероятностей и нормального распределения для мембран от распределения диаметров пор выглядят следующим образом.
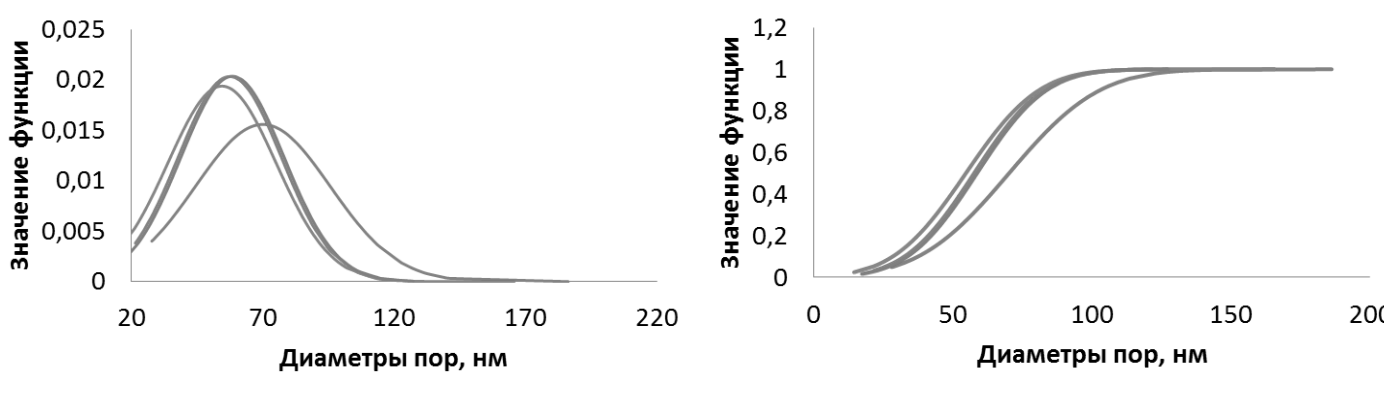

—УАМ-50 —УАМ-100 -УПМ-К —УПМ-100

a

-УАМ-50 —УАМ-100 —УПМ-К -УПМ-100

б

Рис. 4. Функции плотности распределения вероятностей (а) и нормального распределения (б) для мембран УАМ-50, УАМ-100, УПМ-К, УПМ-100 от распределения диаметров пор

Fig. 4. Probability density distribution (a) and normal distribution (b) functions for UAM-50, UAM-100, UPM-K, UPM-100 membranes of the distribution of pore diameters

Согласно утверждению гипотезы о соответствии эмпирического распределения нормальному закону, является необходимым исследование воспроизводимости методики, т.е. определяется возможность исследования первоочередных параметров процесса - среднего арифметического значения и среднего квадратичного отклонения [26]:

$$
f(x)=\frac{1}{\sigma \sqrt{2 \pi}} e^{-\frac{(x-\mu)^{2}}{2 \sigma^{2}}}
$$

где $\mu$ - центр распределения (среднее значение); $\sigma$-разброс распределения (среднее квадратичное отклонение).

В эмпирической форме функция нормального распределения для мембран УАМ-50, УАМ-100, УПМ-К, УПМ-100 от распределения диаметров пор рассчитывается по формулам (6) - (9) соответственно:

$$
\begin{aligned}
& y=\frac{1}{1,97 \sqrt{2 \pi}} e^{\frac{(x-70)^{2}}{2 \cdot 1,97^{2}}} ; \\
& y=\frac{1}{1,44 \sqrt{2 \pi}} e^{\frac{(x-58)^{2}}{2 \cdot 1,44^{2}}} ;
\end{aligned}
$$




$$
\begin{gathered}
y=\frac{1}{1,21 \sqrt{2 \pi}} e^{\frac{(x-54)^{2}}{2 \cdot 1,21^{2}}} \\
y=\frac{1}{1,33 \sqrt{2 \pi}} e^{\frac{(x-59)^{2}}{2 \cdot 1,33^{2}}} .
\end{gathered}
$$

Таким образом, можно с уверенностью утверждать, что ультрафильтрационная мембрана УАМ-50 имеет больший средний диаметр пор по сравнению с УАМ-100, УПМ-К, УПМ-100, отличающиеся практически в 1,2-1,3 раза соответственно, что влияет на проницаемые свойства исследуемых мембран и их задерживающую способность.

\section{Заключение}

В результате проведенных экспериментальных исследований методом электронной микроскопии, стандартных средств обработки данных Microsoft Excel 2010, AutoCad 2018 установлено, что размерный ряд диаметров пор на поверхности мембран УАМ-50, УАМ-100, УПМ-К, УПМ-100 соответствует закону нормального распределения, для которого получены эмпирические выражения, при этом средний диаметр пор мембран УАМ-50, УАМ-100, УПМ-К, УПМ-100 находится в интервале от 54 до 70 нм.

Полученные данные по размерам, распределению пор по диаметрам на поверхности исследуемых ультрафильтрационных мембран УАМ-50, УАМ-100, УПМ-К, УПМ-100 и полученные эмпирические выражения могут использоваться для оценки проницаемости и задерживающей способности пористых мембран.

\section{Список литературы}

1. Ulbricht M. // Polymer. 2006. Vol. 47. I. 7. В.Ф. Селеменев, Т.А. Завьялова. Воронеж: pp. 2217-2262.

2. She Q., Wang R., Fane A.G., Tang C.Y.// изд. ВГУ. 1989. 208 с.

10. Яцев А.М., Акберова Э.М., Голева Е.А., Journal of Membrane Science. 2016. Vol. 499. pp. 201-233.

3. Jhaveri J.H., Murthy Z.V.P. // Desalination. 2016. Vol. 379. pp. 137-154.

Васильева В.И. и др. // Сорбиионные и хроматографические прочессы. 2017. Т. 17. № 2. С. 313-322.

11. Акберова Э.М., Васильева В.И., Смагин

4. Kotra-Konicka K., Kalbarczyk J., Gac J.M. // Chemical and Process Engineering. 2016. Vol. 37. I. 3. pp. 331-339.

5. Liu M., Xiao C., Hu X. // Desalination. 2012. Vol. 298. pp. 59-66.

6. Лазарев С.И., Головин Ю.М., Лазарев Д.С., Казаков В.Г. и др. // Вестник ТГТУ. 2016. Т. 22. № 1. С. 75-83.

7. Коновалов Д.Н., Ковалев С.В., Лазарев С.И., Луа П. И др. // Вестник ТГТУ. 2019. Т. 25. № 4. С. 612-621.

8. Васильева В.И., Голева Е.А., Селеменев В.Ф., Карпов С.И. и др. // Журнал физической химии. 2019. Т. 93. № 3. С. 428-437.

9. Инфракрасная спектроскопия ионообменных материалов / В.А. Углянская, Г.А. Чикин,

М.А., Костылев Д.В. // Сорбиионные и хроматографические проиессы. 2019. Т. 19. № 4. C. $434-442$.

12. Sun W., Chen T., Chen C., Li J. // Journal of Membrane Science. 2007. Vol. 305. I. 1-2. 33. 93-102.

13. Первов А.Г., Андрианов А.П. // Сантехника. 2006. № 5. С. 12-20.

14. Arkhangelsky E., Duek A., Gitis V. // Journal of Membrane Science. 2012. Vol. 394-395. pp. 89-97.

15. Chan Q., Entezarian M., Zhou J., Osterloh R. et al. // Journal of Membrane Science. 2020. Vol. 599. pp. 117822-117825.

16. Fane A.G., Fell C.J.D., Waters A.G. // Journal of Membrane Science. 1981. Vol. 9. I. 3. pp. 245-262. 
17. Sainia B., Khuntiab S., Sinha M.K. // Journal of Membrane Science. 2019. Vol. 572. pp. 184-197.

18. Siddiqui M.U., Arif A.F.M., Bashmal S. // Membranes (Basel). 2016. Vol. 6(3). pp. 40-53.

19. Magueijo V., Semião V., Norberta de Pinho M. // Materials Science Forum. 2006. Vol. 514516. pp. 1483-1487.

20. Myra N., Razali R., Idris A., Yusof K.M. // Jurnal Teknologi. 2008. Vol. 49(F) pp. 229-235.

21. Ibrahim M.Z., Norashikin M.Z. // Journal of Nanoscience and Nanotechnology. 2010. Vol. 10. No 9.. pp. 6211-6215.

22. Cherkasov A.N. // Separation Science and Technology. 2005. Vol. 40. I. 14. pp. 2775-2801.
23. Мембраны, фильтрующие элементы, мембранные технологии: каталог. - Владимир: ЗАО НТЦ «Владипор». 2007. 22 с.

24. Солонин И.С. Применение математической статистики в технологии машиностроения. Свердловск: Средне-Уральское книжное издательство. 1966. 200 с.

25. Колзунова Л.Г., Гребень В.П., Супонина А.П. // Электрохимия. 2003. Т. 39. № 12. С. 1452-1461.

26. Lazarev S.I., Kovaleva O.A., Golovin Y.M., Ryzhkin V.Y. // Сорбиионные и хроматографические прочессы. 2018. Т. 18. № 1. pp. 83-92.

\title{
Electronic-microscopic researches of por distribution by diameters on the sorption surface of UAM-50, UAM-100, UPM-K, UPM-100 ultra-filtration membranes
}

\author{
(C) 2020 Lazarev S.I. ${ }^{1}$, Kovalev S.V. ${ }^{1}$, Konovalov D.N. ${ }^{1}$, Ryzhkin V.YU. ${ }^{1}$, \\ Polyansky K.K. ${ }^{2}$, Korenkov V.V. ${ }^{3}$, Khorokhorina I.V. ${ }^{1}$, Kovaleva O.A. ${ }^{1}$ \\ ${ }^{1}$ Tambov State Technical University, Tambov \\ ${ }^{2}$ Voronezh branch of Plekhanov Russian University of Economics, Voronezh \\ ${ }^{3}$ Tambov State University. G.R. Derzhavina, Tambov
}

The study substantiates the relevance of the topic under investigation and qualifies its place among other publications regarding the porosity of membranes. Based on the literature review and the formulated aim of the study, the types of ultrafiltration membranes were selected and their main characteristics (operating pressure, specific solvent flux, retention coefficient, operating $\mathrm{pH}$ range, maximum temperature) were presented. The electron microscopic study of the pore distribution according to the diameter on the surface of UAM-50, UAM-100, UPM-K, and UPM-100 ultrafiltration membranes was used in the study. This study included fixing the image of the membrane surface using an electron microscope, transferring it to the AutoCad 2018 automated design environment, visual selection of four uniform zones with an area of $1 \cdot 10^{6} \mathrm{~nm}^{2}$ with a description of the pore circumference using the program, data export with the calculation of radial dimensions on the surface (active) layer of the membrane in $\mathrm{nm}$. The main characteristics (diameter, area of each element) were determined using the functions of AutoCad 2018, then the contamination factor was determined. For the estimation of the measurement error for the pore size we analysed 5 electronic images that were obtained for different areas of the surface of the membrane under study. The procedure for processing each electronic image was repeated 10 times. As a result of statistical processing of the results, the relative standard deviation did not exceed 0.1. The standard deviation and the average pore diameter were determined using the descriptive statistics function of Microsoft Excel 2010. The histograms, graphs of the probability density function, and normal distribution for the studied membranes by the pore diameter distribution were plotted using Microsoft Excel 2010.

It was established that the distribution of pores by diameter on the surface of the active layer of the studied ultrafiltration membranes was described by the normal distribution law (Gaussian law). Empirical expressions for the Gaussian distribution of pores by diameters were obtained. Analysis of experimental studies by electron microscopy, standard data processing using Microsoft Excel 2010, and AutoCad 2018 showed that for UAM-50, UAM-100, UPM-K, UPM-100 membranes the average pore diameter on the surface was in the range from 54 to $70 \mathrm{~nm}$.

Keywords: diameter of microstructural inhomogeneities, electron microscopic, normal distribution function, technique, histogram. 


\section{References}

1. Ulbricht M., Polymer, 2006, Vol. 47, I. 7, pp. 2217-2262.

2. She Q., Wang R., Fane A.G., Tang C.Y., Journal of Membrane Science, 2016, Vol. 499, pp. 201-233.

3. Jhaveri J. H., Murthy Z.V.P., Desalination, 2016, Vol. 379, pp. 137-154.

4. Kotra-Konicka K., Kalbarczyk J., Gac J.M., Chemical and Process Engineering, 2016, Vol. 37, I. 3, pp. 331-339.

5. Liu M., Xiao C., Hu X., Desalination, 2012, Vol. 298, pp. 59-66.

6. Lazarev S.I., Golovin Yu.M., Lazarev D.S., Kazakov V.G. et al., Vestnik TGTU, 2016, Vol. 22, No 1, pp. 75-83.

7. Konovalov D.N., Kovalev S.V., Lazarev S.I., Lua $\mathrm{P}$ et al., Vestnik TGTU, 2019, Vol. 25, No 4, pp. 612-621.

8. Vasil'eva V.I., Goleva E.A., Selemenev V.F., Karpov S.I. et al., Russian Journal of Physical Chemistry A, 2019, Vol. 93, No 3, pp. 542-550.

9. Infrakrasnaya spektroskopiya ionoobmennyh materialov, V.A. Uglyanskaya, G.A. Chikin, V.F. Selemenev, T.A. Zav'yalova, Voronezh, VGU, 1989, 208 p.

10. Yatcev A.M., Akberova E.M., Goleva E.A., Vasil'eva V.I. et al., Sorbtsionnye $i$ khromatograficheskie protsessy, 2017, Vol. 17, No 2, pp. 313-322.

11. Akberova E.M., Vasil'eva V.I., Smagin M.A., Kostylev D.V., orbtsionnye i khromatograficheskie protsessy, 2019, Vol. 19, No 4, pp. 434-442.

12. Sun W., Chen T., Chen C., Li J., Journal of Membrane Science, 2007, Vol. 305, I. 1-2, pp. 93-102.

13. Pervov A.G., Andrianov A.P., Santekhnika, 2006, No 5, pp. 12-20.

Лазарев Сергей Иванович - профессор кафедры «Механика и инженерная графика», д.т.н., Тамбовский государственный технический университет, Тамбов

Ковалев Сергей Владимирович - профессор кафедры «Механика и инженерная графика», д.т.н., Тамбовский государственный технический университет, Тамбов

Коновалов Дмитрий Николаевич - доцент кафедры «Техника и технологии автомобильного транспорта», к.т.н., Тамбовский государственный технический университет, Тамбов
14. Arkhangelsky E., Duek A., Gitis V., Journal of Membrane Science, 2012, Vol. 394-395, pp. 89-97.

15. Chan Q., Entezarian M., Zhou J., Osterloh R. et al., Journal of Membrane Science, 2020, Vol. 599, pp. 117822-117825.

16. Fane A.G., Fell C.J.D., Waters A.G., Journal of Membrane Science, 1981, Vol. 9, I. 3, pp. 245-262.

17. Sainia B., Khuntiab S., Sinha M.K., Journal of Membrane Science, 2019, Vol. 572, pp. 184197.

18. Siddiqui M.U., Arif A.F.M., Bashmal S., Membranes (Basel), 2016, Vol. 6(3), pp. 40-53.

19. Magueijo V., Semião V., Norberta de Pinho M., Materials Science Forum, 2006, Vol. 514516, pp. 1483-1487.

20. Myra N., Razali R., Idris A., Yusof K.M., Jurnal Teknologii, 2008, Vol. 49(F), pp. 229235.

21. Ibrahim M.Z., Norashikin M.Z., Journal of Nanoscience and Nanotechnology, 2010, Vol. 10, No 9, pp. 6211-6215.

22. Cherkasov A.N., Separation Science and Technology, 2005, Vol. 40, I. 14, pp. 27752801.

23. Membrany, fil'truyushchie 717lement, membrannye tekhnologii: katalog, Vladimir, ZAO NTC «Vladipor», 2007, 22 p.

24. Solonin I.S. Primenenie matematicheskoj statistiki v tehnologii mashinostroenija, Sverdlovsk, Sredne-Ural'skoe knizhnoe izdatel'stvo, 1966, $200 \mathrm{p}$.

25. Kolzunova L.G., Greben V.P., Suponina A.P., Russian Journal of Electrochemistry, 2003, Vol. 39, No 12, pp. 1300-1307.

26. Lazarev S.I., Kovaleva O.A., Golovin Y.M., Ryzhkin V.Y., Sorbtsionnyye i khromatograficheskiye protsessy, 2018, Vol. 18(1), pp. 8392.

Lazarev Sergey I. - professor Department of Mechanics and Engineering Graphics, Doctor of Technical Sciences, Tambov State Technical University, Tambov

Kovalev Sergey V. - professor Department of Mechanics and Engineering Graphics, Doctor of Technical Sciences, Tambov State Technical University, Tambov

Konovalov Dmitriy N. - Associate Professor of the Department of Engineering and Technology of Road Transport, Ph.D., Tambov State Technical University, Tambov 
Рыжкин Владимир Юрьевич - аспирант, Тамбовский государственный технический университет, Тамбов

Полянский Константин Константинович профессор кафедры «Коммерции и товароведения», д.т.н., Воронежский филиал Российского государственного университета им. Плеханова, Воронеж

Кореньков Виктор Васильевич - с.н.с. научнообразовательного центра «Нанотехнологии и наноматериалы», к.ф.-м.н., Тамбовский государственный университет им. Г.Р. Державина, Тамбов

Хорохорина Ирина Владимировна --доцент кафедры «Природопользование и защита окружающей среды», к.т.н., Тамбовский государственный технический университет, Тамбов

Ковалева Ольга Александровна - профессор кафедры «Механика и инженерная графика», д.т.н., Тамбовский государственный технический университет, Тамбов
Ryzhkin Vladimir Yu. -- Student, Tambov State Technical University, Tambov

Polyansky Konstantin K. - Professor, Department of Commerce and Commodity Science, Doctor of Technical Sciences, Voronezh Branch of the Russian State University them. Plekhanova, Voronezh

Korenkov Victor V. - Senior Researcher Scientific and Educational Center «Nanotechnology and Nanomaterials», Ph.D., Tambov State University named after G.R. Derzhavina, Tambov

Khorokhorina Irina V. - Associate Professor of the Department of Nature Management and Environmental Protection, Ph.D., Tambov State Technical University, Tambov

Kovaleva Olga A. - professor Department of Mechanics and Engineering Graphics, Doctor of Technical Sciences, Tambov State Technical University, Tambov 